\title{
The use of morphometric and fractal parameters to assess the effects of 5-fluorouracil, interferon and dexamethasone treatment on colonic anastomosis healing: an experimental study in rats
}

\author{
Katarzyna Łętowska-Andrzejewicz ${ }^{1}$, Anna Torres ${ }^{1}$, Kamil Torres ${ }^{1,2}$, Piotr Dobrowolski ${ }^{3}$, \\ Tomasz Piersiak ${ }^{3}$, Ryszard Maciejewski ${ }^{1,4}$, Antoni Gawron ${ }^{3}$, Grzegorz Staśkiewicz ${ }^{1}$, \\ Zbigniew Plewa ${ }^{5}$
}

${ }^{1}$ Laboratory of Biostructure, Human Anatomy Department, Medical University of Lublin, Poland

${ }^{2}$ Department of General and Oncologic Surgery, District Specialist Hospital, Lublin, Poland

${ }^{3}$ Department of Comparative Anatomy and Anthropology, UMCS, Lublin, Poland

${ }^{4}$ Medical Emergency Department, UITM Rzeszow, Poland

${ }^{5}$ Department of General Surgery, Military Hospital, Lublin, Poland

\begin{abstract}
Adjuvant chemotherapy and steroid therapy have been demonstrated to interfere with the wound healing process. The aim of this study was to evaluate the effects of 5-fluorouracil, interferon, and dexamethasone, on the healing of colon anastomosis by assessing morphometric and fractal parameters of the colonic wall. An experimental anastomosis of the ascending colon was performed in 60 male Wistar rats, which were then randomly assigned to four groups. On the second to sixth post-operative days, the rats were administered 5 -fluorouracil, interferon- $\alpha$, dexamethasone, or $0.9 \% \mathrm{NaCl}$ solution as a control. Macroscopic, histomorphometric and microbiological evaluation was performed in order to assess healing of the anastomosis. In three animals from the dexamethasone group, there was leakage of anastomosis; adhesion formation was highest in the interferon group, and significantly higher than in the control and 5-fluorouracil groups. Histomorphometric parameter alterations were most pronounced on the seventh and fourteenth post-operative days in all treatment groups, with submucosal thickness the most affected parameter. Connective tissue fractal dimension was significantly decreased in those animals treated with interferon and dexamethasone. All three pharmaceutical agents impaired healing of anastomosis, and promoted infection in the anastomosis and skin wound sites. As dexamethasone induced both morphometric and macroscopic alterations, it was considered the most detrimental in this study. (Folia Histochemica et Cytobiologica 2011; Vol. 49, No. 1, pp. 80-89)
\end{abstract}

Key words: colon, anastomosis, healing, 5-fluorouracil, interferon, dexamethasone

\section{Introduction}

The management of patients suffering from gastrointestinal neoplasms usually requires surgical re-

Correspondence address: G. Staśkiewicz,

Department of Human Anatomy, Medical University of Lublin, Jaczewskiego Str. 4, 20-094 Lublin, Poland; tel.: (+ 48) 609522 559; fax: (+ 48 81) 52888 18;

e-mail: grzegorz.staskiewicz@gmail.com section followed by adjuvant chemotherapy. Patients operated upon due to advanced bowel inflammation need a follow-up comprising the administration of systemic steroids [1,2]. Both treatments have been found to have local and systemic adverse effects [3-6]. Since its introduction, 5-fluorouracil has been considered the gold standard therapy for colorectal cancer. It can be administered alone, or in combination with other pharmaceutical agents, including interferon or the newly-developed cisplatin derivative oxaliplatin [7-10]. 
It has been demonstrated that surgery itself stimulates the development of micrometastases and promotes cell proliferation in already existing metastatic sites [11, 12].

Therefore, early or immediate implementation of adjuvant chemotherapy seems to be beneficial in terms of residual disease, liver metastases and recurrence prevention, and has been demonstrated to improve disease outcomes [13, 14]. The literature describing the effects of chemotherapeutics on the healing process is not wholly consistent, but several studies have reported the deleterious influence of 5 -fluorouracil on the integrity of intestinal anastomosis. Post-operative complications have been shown to have a negative impact on long-term outcomes, and enteroanastomosis dehiscence has been observed to worsen prognosis in colorectal cancer patients [15, 16]. Thus, despite its obvious efficacy in tumor treatment, adjuvant therapy is usually postponed until the wound healing process is complete.

Some of the adverse effects of 5-fluorouracil may result from the down-regulation of collagen synthesis, as well as from suppression of local defense mechanisms in the site of anastomosis [17-19]. A similar influence has been attributed to steroid therapy. Systemic steroids have been demonstrated in rat experimental models to inhibit angiogenesis and the synthesis of collagen, to stabilize lysosomal membranes of the leukocytes, and to impede migration of proinflammatory cells [20-22]. Alterations of systemic and local immunity responses evoked by steroid intake may promote the development of pathogenic bacterial flora, something which further slows the healing process $[18,23]$. Even if properly treated, infections may contribute to anastomosis and abdominal wound dehiscence, scar overgrowth and subsequent incisional hernia, requiring additional surgical intervention [24].

Most of the studies which have evaluated the influence of chemotherapeutic and steroid agents on colonic anastomosis healing have concentrated on bursting pressures and collagen content analysis with standard histological methods $[25,26]$.

Our study aimed to evaluate the effects of 5-fluorouracil, interferon, and dexamethasone on the healing of colon anastomosis, using histomorphometric and fractal parameters of the colonic wall, which are objective and quantifiable. Additionally, bacterial flora growth at the site of anastomosis and skin wound was examined. To the best of our knowledge, this is one of the very few studies that have incorporated histomorphometric parameters and fractal dimensions in evaluating the effects on the colonic anastomosis healing process.

\section{Material and methods}

\section{Animals and groups}

The study was performed on 60 male Wistar rats weighing 250-350 grams. In an effort to minimize deaths following surgery, animals were brought to the laboratory seven days before the start of the experiment for adaptation, and to reduce the stress related to transportation. The animals were acclimatized and housed under standard conditions, three to a cage. They were allowed standard rat chow and water $a d$ libitum throughout the study.

The study protocol was approved by the Bioethics Committee of the Medical University of Lublin.

\section{Operating procedure and treatment regimes}

Before the surgery, the animals underwent general anesthesia with nembutal, administered intraperitoneally in a dosage of $50 \mathrm{mg}$ per kilogram body weight. After skin disinfection, a $3 \mathrm{~cm}$ skin and peritoneum fragment was removed using surgical scissors. An excision was performed rather than an incision in order to extend the operative field and to avoid the use of retractors that would additionally traumatize the edges of the wound. The ascending colon was exposed and incised just above the cecum in order to allow an easy identification of the anastomosis area after the surgery. The side to side enteroanastomosis was created using interrupting inverting 5.0 polyglactin sutures. The peritoneum was closed using a continuous absorbable polyglactin 3.0 surgical suture. Closing of the skin incision was performed using interrupted nonabsorbable nylon 5.0 sutures. The animals were then placed in cages for 30 minutes and warmed up. Just after the surgery, the animals were allowed only water with $5 \%$ glucose $(1: 1$ ratio) and solid food was introduced on the second post-operative day. Antibiotic prophylaxis was not applied.

After the surgery, animals were randomly assigned to one of four groups. Animals in the control group ('Group 1') were injected with an isotonic salt solution. Animals in the three remaining groups were injected with 5-fluorouracil ( $20 \mathrm{mg} / \mathrm{kg} /$ day) ('Group 2 '), interferon- $\alpha$ (45.000 IU/kg/day) ('Group 3') and dexamethasone ( $0.5 \mathrm{mg} /$ day) ('Group 4'). All the substances were administered for five days, starting from the second post-operative day, via intraperitoneal injections. The injections were performed by a single researcher at the same time of day throughout the study. 


\section{Analytical procedures}

The rats were sacrificed using an intraperitoneal injection of thiopental and dissected on either the fourth, seventh or fourteenth day after surgery. Before each dissection, macroscopic evaluation of the scar was performed. Samples for microbiological examination were collected from the skin wounds and anastomosis sites of animals which presented with the signs of commencing or ongoing infection. Reopening of the post-operative wound was made with sterile scissors, and the enteroanastomosis area was examined, taking particular care to maintain the sterility of the area. Initially, the anastomosis was evaluated macroscopically for the presence of dehiscence, abscesses, adhesions, fistulae and symptoms of peritonitis. A scale used previously by Van der Ham in a similar study was applied for that evaluation [27]. According to that scale, zero adhesions between anastomosis and the surrounding structures were graded 0 ; minimal adhesions between anastomosis and greater omentum were graded 1 ; larger adhesions between anastomosis and greater omentum and between the anastomised parts and the small intestine loop were graded 2; and large adhesions and abscesses around the anastomosis were graded 3 .

Areas suspected of having infection were sampled for microbiological examination and cultures were immediately set on a standard medium. After macroscopic inspection, a $3 \mathrm{~cm}$ fragment of the colon with anastomosis site was resected and the leak-tightness of the anastomosis was evaluated. Afterwards, a fragment of tissue containing anastomosis was incubated in a $4 \%$ formaldehyde solution. After being embedded in paraffin, specimens were cut in a microtome (Microm HM 360) into $5 \mu \mathrm{m}$ sections. Twenty sections were obtained from each specimen (at $20 \mu \mathrm{m}$ intervals after each series of five sections). Staining with hematoxylin and eosin was performed afterwards. Microscopic images were obtained using a Nikon Eclipse E800-light microscope and a Nikon D70 digital camera, and underwent computerized planimetric analysis using Image ${ }^{\circledR}$ v. $1.37 \mathrm{a}$, an image processing and analysis software. The following parameters were considered in the evaluation: total crypt number per millimeter, active crypt number per millimeter, inactive crypt number per millimeter, mucosa thickness, submucosa thickness, myenteron thickness, mucosa fractal dimension (according to the box-counting method) and connective tissue fractal dimension (using a UV microscope). White blood cells count was also evaluated in tail vein blood samples obtained on the fourth, seventh and fourteenth post-operative days.

\section{Statistical analysis}

Continuous variables were presented as mean values with standard deviation and $95 \%$ confidence interval. Differences between the groups were evaluated using one-way analysis of variance (ANOVA), Duncan's multiple comparison test, and nonparametric Mann-Whitney U test. P values of $<0.05$ were considered to be significant. The statistical analysis was performed using Microsoft Statistica 6.0 software.

The results of a comparative analysis are presented on boxplot graphs representing mean value, standard error of the mean (boxes) and confidence interval of the mean (whiskers).

\section{Results}

Four animals (two from the dexamethasone group and two from the interferon group) died after the surgery. Macroscopic evaluation of the abdominal cavity performed during the post mortem section did not reveal any significant deviations. Neither symptoms of peritonitis, post-operative adhesions nor abscesses were observed. Therefore it was assumed that the death of the animals was the result either of the medicine used to induce anesthesia or stress connected with surgical intervention. The four animals were excluded from further analysis.

\section{Macroscopic evaluation}

Macroscopic inspection of the anastomosis and the post-operative skin wound revealed the presence of an inflammatory process in 32 rats. Nevertheless, neither a perforation, nor an intra-abdominal abscess nor an anastomosis rupture was observed in any of the groups. Evaluation of the leak-tightness of the anastomoses demonstrated leakage in three of the cases treated with dexamethasone. This occurred on the seventh post-operative day. No leaks were present in other study groups.

The average adhesion formations on the fourth, seventh and fourteenth post-operative days are presented in Table 1. Adhesion formation average scores differed between the groups on the fourth post-operative day, and were highest in the interferon group.

\section{Histomorphometric evaluation}

Changes in the histomorphometric parameters were observed in both the control and the treated groups. But more intense alterations were seen in the treated groups. 
Table 1. Average adhesion formations on the fourth, seventh and fourteenth post-operative days

\begin{tabular}{|l|c|c|c|c|}
\hline $\begin{array}{l}\text { Day } \\
\text { of } \\
\text { experiment }\end{array}$ & Control & 5-fluorouracil & Interferon & Dexamethasone \\
\hline $4^{\text {th }}$ & 1 & 2 & 3 & 2.5 \\
\hline $7^{\text {th }}$ & 1 & 1 & 2 & 1.3 \\
\hline $14^{\text {th }}$ & 1 & 1 & 1.5 & 1 \\
\hline
\end{tabular}

Analysis of the histomorphometric parameters performed in samples collected on the fourth post-operative day revealed a significant decrease in submucosal thickness (Figure 1) and an increase in inactive crypt number in all treated animals. The latter parameter was highest in the 5-fluorouracil group and was accompanied by increased mucosal and myenteron thickness compared to the control group. On the seventh post-operative day, a further reduction in the submucosal diameter was observed in the 5-fluorouracil group. Increases in active, and decreases in inactive, crypt numbers were observed in those animals treated with dexamethasone and 5-fluorouracil on that day also. Additionally, fractal dimension of the connective tissue was reduced in the interferon-treated and the dexamethasonetreated groups (Figure 2), whereas the mucosa fractal dimension was increased in those groups (Figure 3). On the fourteenth post-operative day, significant decreases of submucosa and myenteron thicknesses, as well as reduced mucosa fractal dimension, were present in all groups compared to controls (Figures $4 \mathrm{~A}-\mathrm{C}$ ).

The highest reduction of myenteron thickness was observed in the interferon-treated animals, as was the fractal dimension of the mucous membrane. The greatest reduction of submucosa thickness was in the group treated with 5-fluorouracil.

When the parameters were compared within the groups according to time, it was found that a decrease of almost all studied morphometric parameters occurred on the seventh post-operative day in the controls. Thereafter, on the fourteenth day after surgery, the parameters regained values similar to those observed on the fourth day. Values of the morphometric parameters obtained on fourth, seventh and fourteenth day of the experiment in the group treated with dexamethasone were not significantly different. A different sequel was observed in the groups treated with interferon and 5-fluorouracil, in which particular parameters decreased progressively. A progressive decrease of mucosa, submucosa and myenteron thickness was observed in the 5-fluorouracil group (Figures 5A-C), whereas the interferon group witnessed a progressive

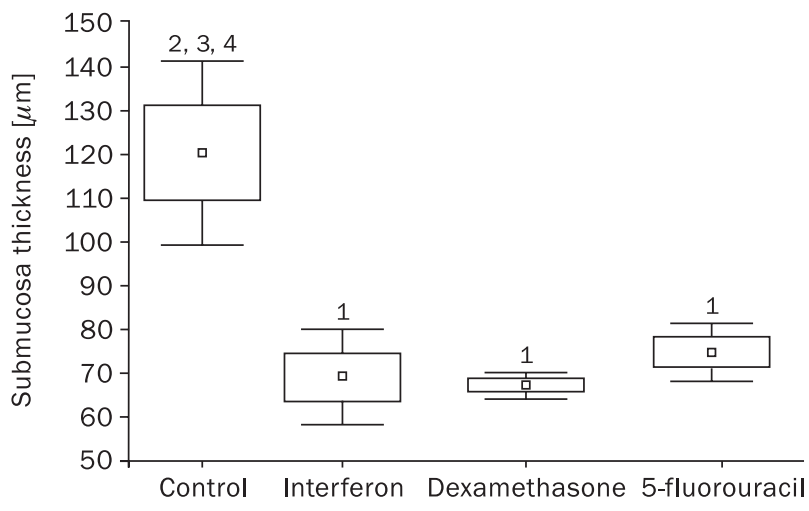

Figure 1. Thickness of submucosa on the fourth postoperative day. Numbers above boxplots indicate statistically significant differences ( $\mathrm{p}<0.05)$ : 1 . vs. control group; 2. vs. interferon group; 3. vs. dexamethasone group; 4. vs. 5-fluorouracil group

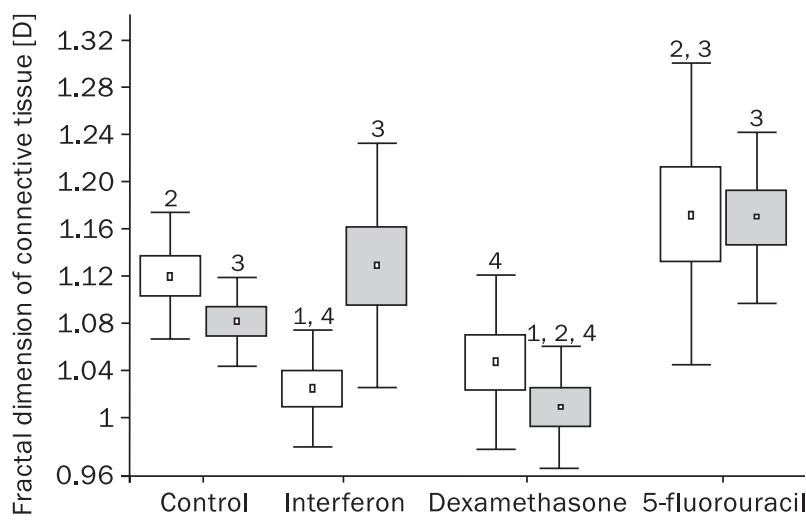

Figure 2. Fractal dimensions of connective tissue of large intestine on seventh (white boxes) and fourteenth (grey boxes) post-operative days. Numbers above boxplots indicate statistically significant differences $(\mathrm{p}<0.05)$ : 1 . vs. control group; 2 . vs. interferon group; 3 . vs. dexamethasone group; 4. vs. 5-fluorouracil group

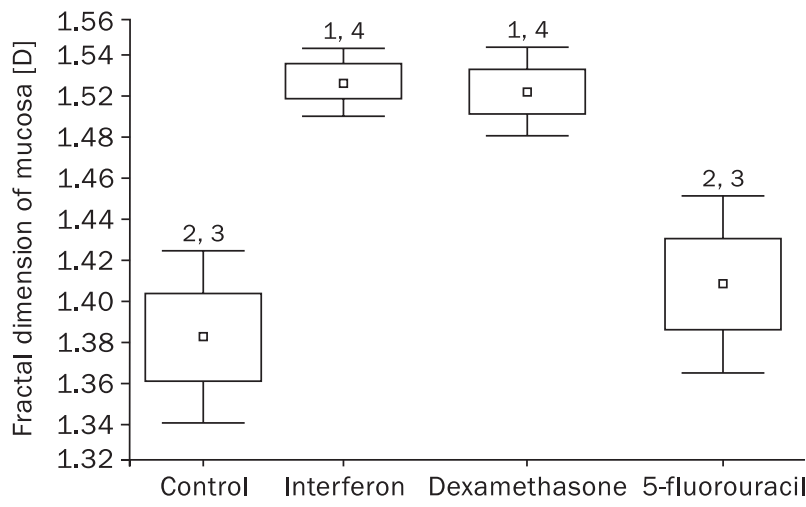

Figure 3. Fractal dimensions of mucosa of large intestine on the seventh post-operative day. Numbers above boxplots indicate statistically significant differences $(p<0.05)$ : 1 . vs. control group; 2 . vs. interferon group; 3 . vs. dexamethasone group; 4. vs. 5-fluorouracil group 
A

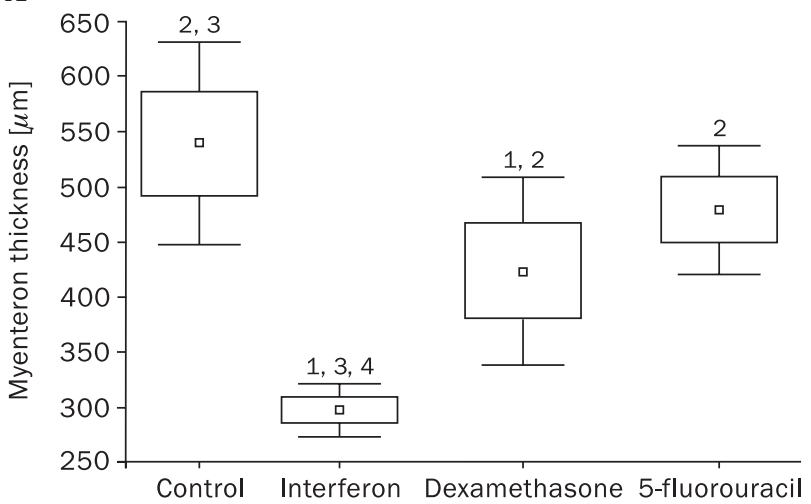

C

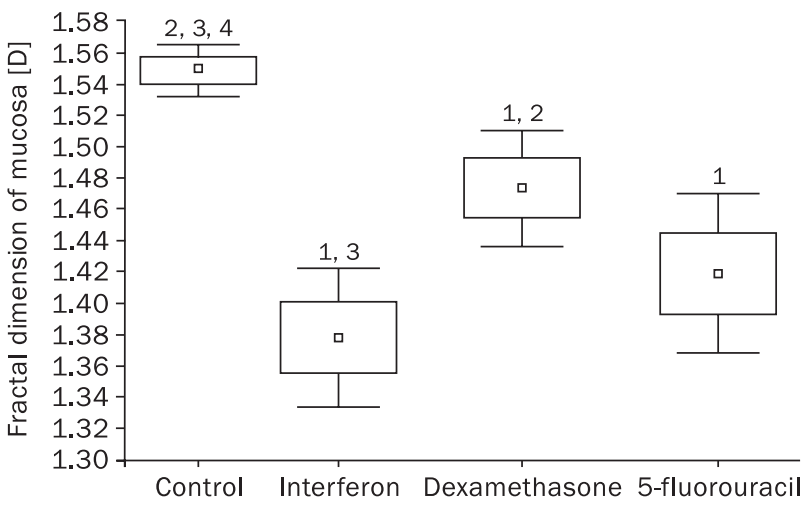

B

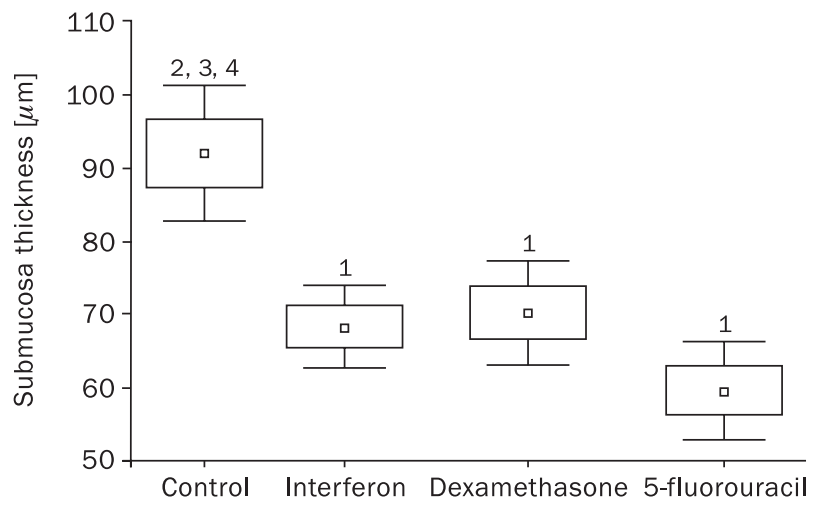

Figure 4. Parameters measured on fourteenth postoperative day: A. myenteron thickness; B. submucosa thickness; C. fractal dimension of mucosa. Numbers above boxplots indicate statistically significant differences ( $p<0.05$ ): 1. vs. control group; 2 . vs. interferon group; 3 . vs. dexamethasone group; 4. vs. 5-fluorouracil group

A

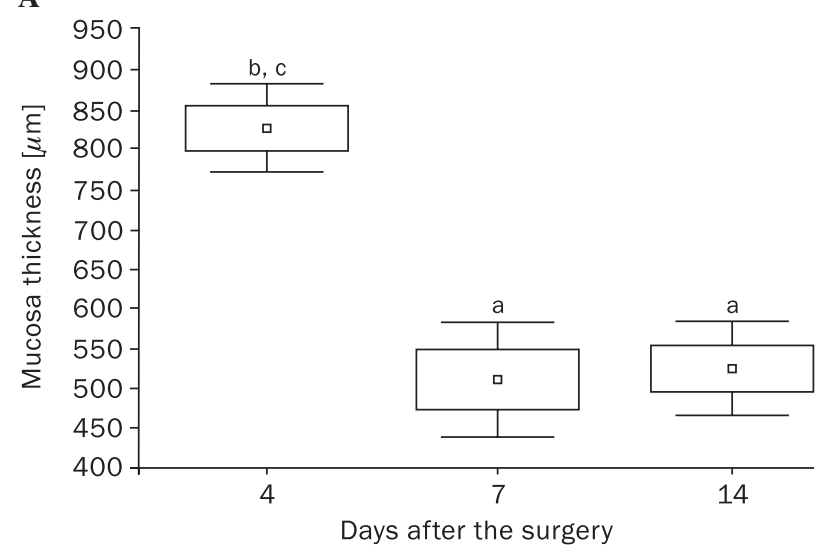

C

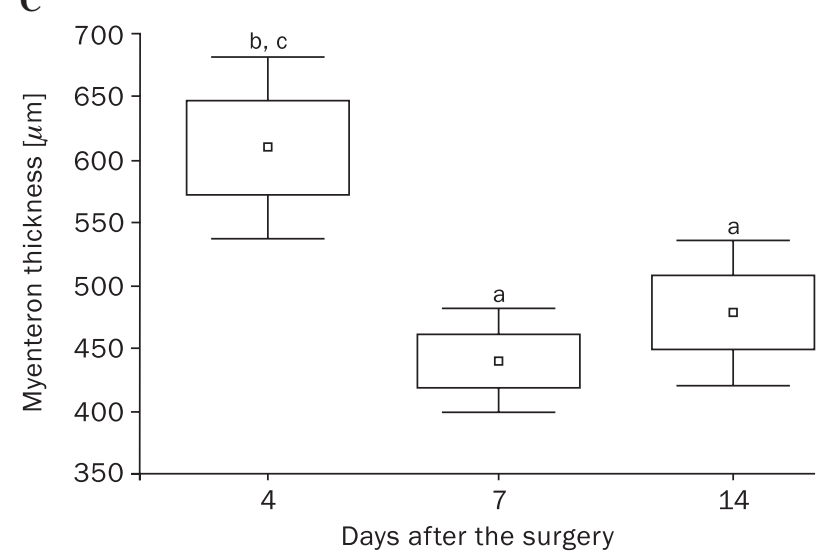

B

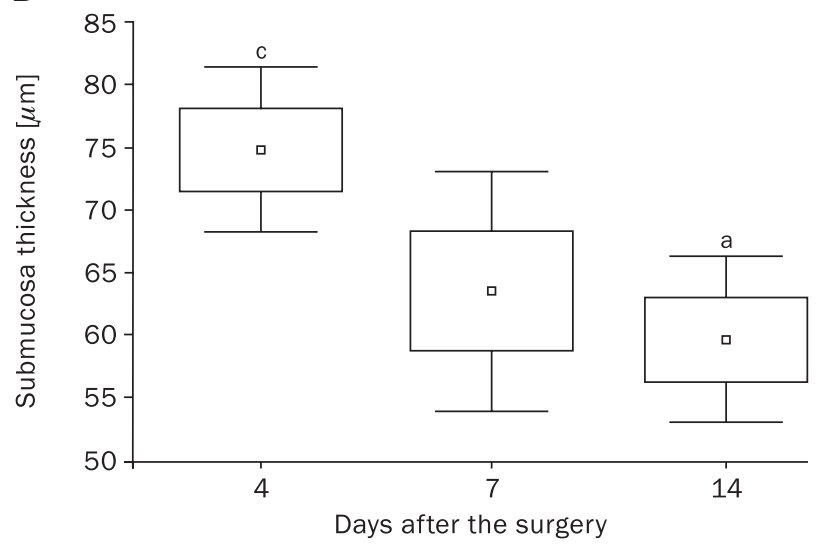

Figure 5. Changes of parameters on fourth, seventh and fourteenth post-operative day in the 5-fluorouracil group: A. thickness of mucosa; B. thickness of submucosa; C. myenteron thickness. Letters above boxplots indicate statistically significant differences $(p<0.05)$ : a. vs. fourth post-operative day; b. vs. seventh post-operative day; c. vs. fourteenth post-operative day 
A

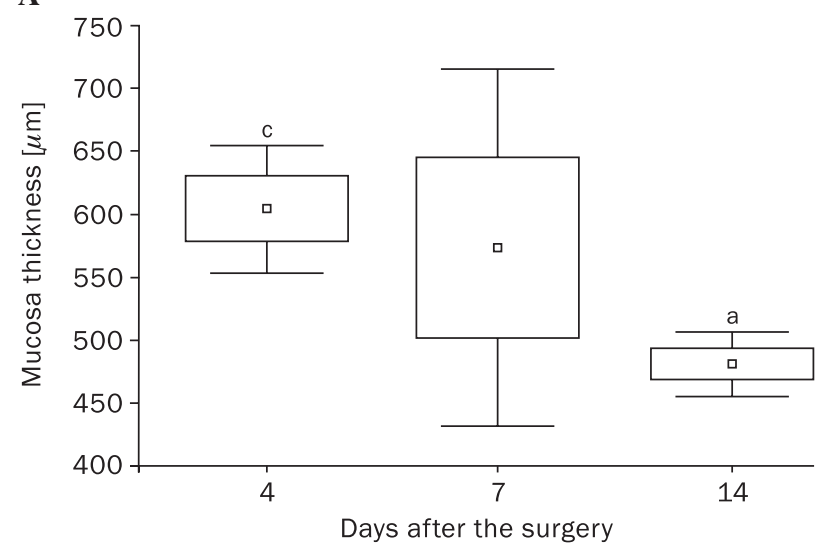

B

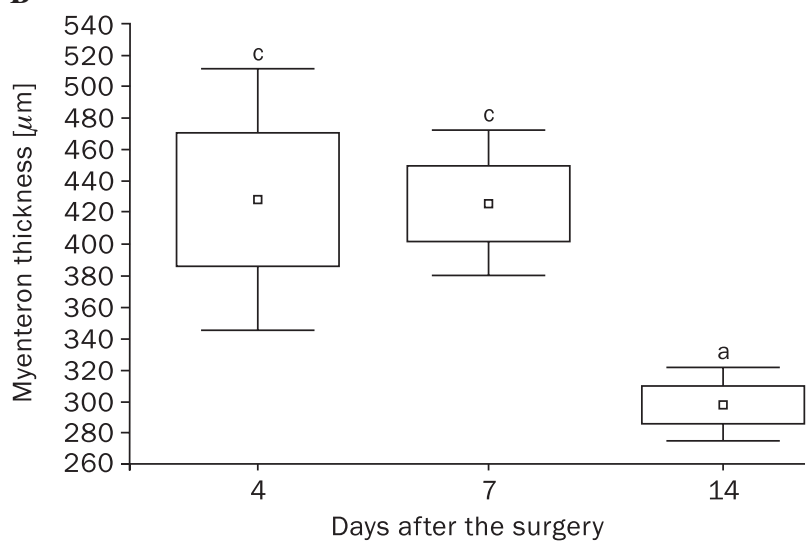

Figure 6. Changes of parameters on fourth, seventh and fourteenth post-operative days in the interferon group: A. thickness of mucosa; B. myenteron thickness. Letters above boxplots indicate statistically significant differences $(p<0.05)$ : a. vs. fourth post-operative day; b. vs. seventh post-operative day; c. vs. fourteenth post-operative day

reduction of mucosa (Figure 6A) and myenteron thickness (Figure 6B), as well as mucosa fractal dimension. At the same time, inactive crypt number gradually increased in interferon-treated animals.

\section{Connective tissue fractal dimension changes}

Connective tissue fractal dimension was significantly impaired in the interferon group on the seventh post-operative day compared to controls; it was also reduced on the fourteenth post-operative day in animals treated with dexamethasone (Figure 2). Additional analysis revealed that in animals treated with interferon, it was significantly increased on the fourteenth compared to the fourth post-operative day. No other statistically relevant differences were encountered regarding that parameter.

\section{Microbiological cultures}

Collection of specimens for microbiological examination was performed in 32 animals, which presented symptoms of intraperitoneal or skin wound infection. On the fourth post-operative day, samples were collected from eight animals, two in each group. On the seventh day, a group of 14 animals qualified for microbiological examination, six of which belonged to the dexamethasone group, four to the interferon group, and two each to the 5-fluorouracil and control groups. Of the ten animals diagnosed with infection on the fourteenth post-operative day, four belonged to the control group, and two to each of the pharmacologically managed groups.

Bacterial growth was observed exclusively in samples collected on the fourth post-operative day from the treated animals. Specimens obtained from the control group did not reveal the presence of any pathogens. The highest variety of pathogens was observed in the interferon group. Microbiological culture results are presented in Table 2.

\section{White blood cell count (WBC) evaluation}

The lowest WBC was observed in the 5-fluorouracil group on the seventh post-operative day, and the highest number of white blood cells occurred in the dexamethasone-treated animals on the fourteenth postoperative day. Lymphocytosis (80-90\%) was present in all experimental animals, and was highest in the control group on the fourteenth post-operative day.

\section{Discussion}

For many years, 5-fluorouracil has been used in adjuvant therapy of colorectal cancer, and it is still considered the gold standard in the post-operative management of this neoplasm $[1,8]$. The utility of interferons has been also demonstrated for the treatment of colon carcinoma in a number of studies, as agents that can be administered alone or in combination with chemotherapeutics $[9,28,29]$. Dexamethasone was incorporated in our study protocol because steroids are commonly used in the post-operative management of patients undergoing bowel resection due to inflammatory bowel diseases $[2,3]$. The immediate or very early implementation of adjuvant treatment is much more efficacious in terms of survival rates and metastases prevention compared to delayed chemotherapy.

However, it is commonly withheld until the wound healing process is completed in order to prevent post- 
Table 2. Microbiological culture results in study groups on the fourth post-operative day

\begin{tabular}{|c|c|c|c|}
\hline No. & Group & Enteroanastomosis culture & Skin wound culture \\
\hline 1 & 5 -fluorouracil & Escherichia coli, Proteus species & None \\
\hline 2 & 5 -fluorouracil & Escherichia coli, MRSA & Escherichia coli \\
\hline 3 & Interferon & Escherichia coli, Proteus species, MRSA & MRSA, Pseudomonas aeruginosa \\
\hline 4 & Interferon & Escherichia coli, MRSA & Escherichia coli \\
\hline 5 & Dexamethasone & Escherichia coli & None \\
\hline 6 & Dexamethasone & None & None \\
\hline 7 & Control & None & None \\
\hline 8 & Control &
\end{tabular}

-operative complications, which have been shown to worsen patients' prognosis in colorectal cancer [12, 15, 16]. Several studies have evaluated the effects of different chemotherapeutics on anastomosis healing. The methodology used in most of those studies relied on the analysis of the macroscopic status of the anastomosis. Additionally, bursting strength, intensity of local inflammation, and collagen content have been used by some authors $[25,26]$. The results of those studies are not completely concordant.

In our study, we used histomorphometric and fractal parameters. In addition, microbiological tests were performed.

Histomorphometric analysis enables objective and quantifiable assessment of the organs' structure [30]. Fractal dimension is a parameter which reflects the complexity and irregularity of studied organs [31]. Tissues undergoing remodeling or modification due to exogenous or endogenous factors are characterized by an increased fractal dimension. Morphometric parameters and fractal geometry have been used to distinguish benign from malignant lesions, to measure tumor infiltrative margins, to assess angiogenesis and distribution of collagen in tissues. Therefore we considered them to be useful tools for evaluating the enteroanastomosis healing process [32-34].

A combination of a reliable experimental model and a method of analysis that enables objectivity and provides high accuracy and uniformity in interpreting the results might shed fresh light on the structural changes responsible for the functional impairment of the anastomosis.

Analysis of the morphometric changes that occurred in the control group indicated that the surgery itself evoked remodeling of the tissue within the anastomosis; in particular, submucosa thickness and the number of active and inactive crypts were reduced. These results align with the study by Simmy et al. who observed alterations in the enterocyte population, crypt cell migration and the length of intestine villi as effects of surgical intestine manipulation [35]. Nevertheless, the alterations of morphometric parameters observed in our study were much more significant in the groups submitted to pharmacological treatment. Administration of 5-fluorouracil and interferon evoked a progressive decrease of the mucosal, submucosal, and myenteric thicknesses, plus a significant reduction of the mucosa fractal dimension. Treatment with dexamethasone was also connected with a marked reduction in the submucosal and myenteric thicknesses, as well as with the decreased mucosa fractal dimension that occurred on the fourteenth post-operative day. A similar negative influence was observed by Kanellos et al., whose histological examination of the anastomoses influenced by 5-fluorouracil treatment revealed an increased rupture rate as well as necrosis and inflammation [36]. Conversely to our results, they did not find interferon to exert any negative effects on the healing process. In fact, on the contrary, treatment with that immunomodulator was connected with increased fibroblast proliferation and collagen synthesis, whereas in our study interferon administration resulted in a significant decrease in the connective tissue fractal dimension, a feature that reflected restoration of the connective tissue structure [36].

Results which seemed to confirm our observation were, however, obtained by Fu et al., who observed impairment of skin wound healing and decreased deposition of new collagen in a rat model of incised skin wound [37]. Deterioration of the morphometric features which was caused by 5-fluorouracil and interferon did not correlate with increased frequency of the anastomotic leakage in our study, which was consistent with the results obtained by Hillan et al. [38]. However, anastomotic leakage occurred in three animals treated with dexamethasone.

The adverse effects of 5-fluorouracil on the healing of colon anastomosis have also been confirmed by other authors. Ersoy et al. compared the influence 
of 5-fluorouracil and oxaliplatin on the colon anastomosis healing process using the bursting pressure protocol with the assessment of hydroxyproline content in the site of anastomosis. They found 5-fluorouracil to negatively influence anastomosis status and to exert significantly more detrimental effects compared to oxaliplatin. In concordance with our results, Ersoy et al. did not find any significant influence of 5-fluorouracil on the connective tissue remodeling reflected as collagen content changes [39]. The detrimental effects of 5-fluorouracil have also been demonstrated by other researchers [26, 40, 41]. In the study by Graf et al., it was found to significantly impair anastomosis healing [40]. Another study (by the same research group) revealed detrimental effects of 5-fluorouracil on collagen synthesis, which was evident on the seventh day after formation of anastomosis [41]. Weiber et al. investigated the relation between the timing of 5-fluorouracil treatment introduction and anastomosis status. Their results showed that delayed treatment resulted in an uncomplicated healing process, whereas beginning the regime on the day of surgery was connected with detrimental effects on anastomosis status [42]. Similar observations were obtained in the studies by Kanellos et al. who compared early and delayed combined treatment with 5-fluorouracil and interferon or folinic acid [43, 44].

In the studies performed by Weiber at al. and Kanellos et al., delayed therapy cycles begun on the third and fourth days after surgery, respectively, were connected with significantly less detrimental influence on the healing process. Early treatment was introduced on the day of surgery in those studies and resulted in visible impairment of the anastomosis status $[42,44]$. In our study, treatment began on the second post-operative day. That could explain why morphometric changes observed in 5-fluorouracil-treated animals did not correlate with macroscopically visible impairment of the anastomosis.

Although many studies have shown the negative effects of 5-fluorouracil on the healing of colon anastomosis, the literature concerning that issue is not entirely consistent. Contrary to the aforementioned studies, Nayci et al. did not reveal impaired anastomosis healing during treatment with 5-fluorouracil [45]. Similar results were also obtained by Stoop et al., who did not observe any influence of such treatment on a bursting pressure of anastomosis [46]. No bursting pressure alterations were present in a study performed by el-Malt et al. either. However, similarly to our observation, the authors reported the presence of histological changes at the anastomotic site in the groups treated with 5-fluorouracil [47]. A study performed by Kuzu et al. investigated the influence of 5-fluorouracil administered before surgery and did not observe any differences in the anastomosis healing between treated and control groups [48]. The only alteration found in that study was a significant decrease in the hydroxyproline content within the anastomoses of the treated animals [48].

A number of studies have shown steroids to exert detrimental effects on the wound healing process. Deterioration of the healing process evoked by steroids is based on several mechanisms, including inhibition of the local inflammatory response, inadequate formation of granulation tissue, and improper metabolism of the connective tissue which results from the impairment of the collagen synthesis and the proliferation of fibroblasts [21, 22, 49]. Our study shows concordant results, as dexamethasone analyzed in our study exerted significant adverse morphometric changes, the most significant being submucosal and myenteric thickness decreases, as well as reduction of mucosa fractal dimension.

Moreover, macroscopic evaluation revealed leakage of the anastomosis in three animals treated with that steroid. Similar results were obtained by Polat et al. and Mantzoros et al., who found not only dexamethasone but also hydrocortisone perioperative treatment to impair anastomosis wound healing [50, 51]. Additionally, Mantzoros et al. observed a correlation between steroid treatment and a decreased deposition of collagen in the anastomotic site [51]. That observation was consistent with our findings, as significantly decreased fractal dimension of the connective tissue was observed in the dexamethasone group on the fourteenth post-operative day. Similar results were also obtained by Eubanks et al., who compared the influence of methyloprednisolone, hydrocortisone and dexamethasone on the colon wound healing process [52]. They did not reveal differences in bursting pressures between studied steroids, but the rate of spontaneous ruptures was significantly higher in the dexamethasone-treated animals [52].

The microbiological characteristics of the anastomotic site have been investigated in only a few studies. Kanellos et al. observed the growth of pathological flora in cases of peritonitis or abscess in animals treated with 5-fluorouracil, or a combination of 5-fluorouracil and interferon, as well as in one animal from the control group. At the same time, the number of animals affected by the pathogenic flora growth was similar in both groups submitted to chemotherapy (seven vs. eight animals) [25]. Concordant results were obtained in our study. The same number of animals from each study group presented with positive microbiological culture, although bacterial spectrum differed. 
The microbiological examination performed by Kanellos et al. revealed the presence of Escherichia coli, Enterococcus, Proteus and Bacteroides spp. [25]. Our study discovered Escherichia coli and Proteus spp. but also methiciline-resistant Staphylococcus aureus $s p$. in animals treated with 5-fluorouracil and interferon. Treatment with dexamethasone was connected with the presence of Escherichia coli spp. and resulted in leakage of the anastomosis in two of three infected animals. Contrary to the results obtained by Kanellos, no pathogens were cultured in the specimens obtained from the control group in our study.

\section{Conclusions}

All three pharmaceutical agents analyzed in our study impaired healing of the anastomoses and promoted infection in the anastomosis and skin wound sites.

Dexamethasone induced both morphometric and macroscopic alterations. Therefore, it was considered the most detrimental in our study. Our results concord with most previous studies, which used macroscopic characteristics and bursting strength to assess the anastomosis healing process.

Therefore, histomorphometric and fractal parameters seem to be useful and valid tools for use in such an experimental setting.

\section{References}

1. Boller AM, Cima RR. Impact of pre- and postoperative multimodality therapy on rectal cancer. J Surg Oncol. 2007;96: 665-670.

2. Andersson P, Olaison G, Bodemar G, Nyström PO, Sjödahl R. Surgery for Crohn colitis over a 28-year period: fewer stomas and the replacement of total colectomy by segmental resection. Scand J Gastroenterol. 2002;37:68-73.

3. Blomqvist P, Feltelius N, Löfberg R, Ekbom A. A 10-year survey of inflammatory bowel diseases-drug therapy, costs and adverse reactions. Aliment Pharmacol Ther. 2001;15:475-481.

4. Hassan I, Cima RR. Quality of life after rectal resection and multimodality therapy. J Surg Oncol. 2007;15; 96:684-692.

5. Dalzell JR, Samuel LM. The spectrum of 5-fluorouracil cardiotoxicity. Anticancer Drugs 2009;20:79-80.

6. Mukai M, Kishima K, Uchiumi F et al. Clinical comparison of QOL and adverse events during postoperative adjuvant chemotherapy in outpatients with node-positive colorectal cancer or gastric cancer. Oncol Rep. 2009;21:1061-1066.

7. Eng C. Toxic effects and their management: daily clinical challenges in the treatment of colorectal cancer. Nat Rev Clin Oncol. 2009;6:207-218.

8. Comella P, Casaretti R, Sandomenico C, Avallone A, Franco L. Role of oxaliplatin in the treatment of colorectal cancer. Ther Clin Risk Manag. 2009;5:229-238.

9. Oosterling SJ, van der Bij GJ, Mels AK et al. Perioperative IFN-alpha to avoid surgically induced immune suppression in colorectal cancer patients. Histol Histopathol. 2006;21:753-760.

10. Turner PK, Houghton JA, Petak I et al. Interferon-gamma pharmacokinetics and pharmacodynamics in patients with colorectal cancer. Cancer Chemother Pharmacol. 2004;53:253-260.
11. Macdonald JS, Astrow AB. Adjuvant therapy of colon cancer. Semin Oncol. 2001;28:30-40.

12. Brodsky JT, Cohen AM. Peritoneal seeding following potentially curative resection of colonic carcinoma: implications for adjuvant therapy. Dis Colon Rectum. 1991;34:723-727.

13. de Bree E, Witkamp AJ, Zoetmulder FA. Intraperitoneal chemotherapy for colorectal cancer. J Surg Oncol. 2002;79:46-61.

14. Sugarbaker PH, Graves T, DeBruijn EA et al. Early postoperative intraperitoneal chemotherapy as an adjuvant therapy to surgery for peritoneal carcinomatosis from gastrointestinal cancer: pharmacological studies. Cancer Res. 1990; 50:5790-5794.

15. Law WL, Choi HK, Lee YM, Ho JW, Seto CL. Anastomotic leakage is associated with poor long-term outcome in patients after curative colorectal resection for malignancy. $J$ Gastrointest Surg. 2007;11:8-15.

16. Law WL, Choi HK, Lee YM, Ho JW. The impact of postoperative complications on long-term outcomes following curative resection for colorectal cancer. Ann Surg Oncol. 2007;14:2559-2566.

17. Graf W, Weiber S, Glimelius B, Jiborn H, PÍhlman L, Zederfeldt B. Influence of 5-fluorouracil and folinic acid on colonic healing: an experimental study in the rat. Br J Surg. 1992;79:825-828.

18. Adachi W, Sugenoya A, Horigome N, Takahashi C, Iida F, Nakayama J. The anti-tumor activity and immunosuppressive effects of 5-fluorouracil suppositories in rectal cancer patients. Surg Today. 1992;22:221-225.

19. Kanellos D, Blouhos K, Pramateftakis MG et al. Effect of 5 -fluorouracil plus interferon on the integrity of colonic anastomoses covering with fibrin glue. World J Surg. 2007;31:186-191.

20. Polat A, Nayci A, Polat G, Aksöyek S. Dexamethasone downregulates endothelial expression of intercellular adhesion molecule and impairs the healing of bowel anastomoses. Eur J Surg. 2002;168:500-506.

21. Patil SD, Papadmitrakopoulos F, Burgess DJ. Concurrent delivery of dexamethasone and VEGF for localized inflammation control and angiogenesis. J Control Release. 2007;117:68-79.

22. Luo JC, Shin VY, Liu ES et al. Dexamethasone delays ulcer healing by inhibition of angiogenesis in rat stomachs. Eur J Pharmacol. 2004;485:275-281.

23. Ziesché E, Scheiermann P Bachmann M et al. Dexamethasone suppresses interleukin-22 associated with bacterial infection in vitro and in vivo. Clin Exp Immunol. 2009; 157:370-376.

24. van Ramshorst GH, Nieuwenhuizen J, Hop WC et al. Abdominal wound dehiscence in adults: development and validation of a risk model. World J Surg. 2010;34:20-27.

25. Kanellos I, Odisseos C, Zaraboukas T, Kavouni A, Galovatsea K, Dadoukis I. Colonic healing after early intraperitoneal administration of 5-fluorouracil and interferon in rats. Int J Colorectal Dis. 1997;12:45-48.

26. Kanellos I, Kavouni A, Zaraboukas T, Odisseos C, Galovatsea K, Dadoukis I. Influence of intraperitoneal 5-fluorouracil plus folinic acid on the healing of colonic anastomoses in rats. Eur Surg Res. 1996;28:374-379.

27. van der Hamm A, Kort W, Weiima I, van den Inhg H, Jeekel H. Effects of antibiotics in fibrin sealant on healing colonic anastomoses in the rat. Br J Surg. 1992;79:525-528.

28. Mitchell MS. Immunotherapy as part of combinations for the treatment of cancer. Int Immunopharmacol. 2003;3: 1051-1059. 
29. Schippinger W, Jagoditsch M, Sorré C et al. Austrian Breast and Colorectal Cancer Study Group. A prospective randomised trial to study the role of levamisole and interferon alfa in an adjuvant therapy with 5-FU for stage III colon cancer. Br J Cancer. 2005;92:1655-1662.

30. de Sousa MV, Priolli DG, Portes AV, Cardinalli IA, Pereira JA Martinez CA. Evaluation by computerized morphometry of histopathological alterations of the colon wall in segments with and without intestinal transit in rats. Acta Cir Bras. 2008;23:417-424.

31. Cross SS. Fractals in pathology. J Pathol. 1997;182:1-8.

32. Dey P. Basic principles and applications of fractal geometry in pathology: a review. Anal Quant Cytol Histol. 2005;27:284-290.

33. Dioguardi N, Franceschini B, Aletti G, Russo C, Grizzi F. Fractal dimension rectified meter for quantification of liver fibrosis and other irregular microscopic objects. Anal Quant Cytol Histol. 2003;25:312-320.

34. Roy HK, Kim YL, Wali RK et al. Spectral markers in preneoplastic intestinal mucosa: an accurate predictor of tumor risk in the MIN mouse. Cancer Epidemiol Biomarkers Prev. 2005;14:1639-1645.

35. Simmy T, Anup R, Prabhu R, Balasubramanian KA. Effect of surgical manipulation of the rat intestine on enterocyte populations. Surgery. 2001;130:479-488.

36. Kanellos I, Odisseos C, Kazantzidou D, Galovatsea K, Zaraboukas T, Dadoukis I. Effect of early versus delayed postoperative injection of 5-fluorouracil plus interferon-alpha on colonic healing. Eur Surg Res. 1998;30:414-418.

37. Fu X, Chang G, Wang Y. Systemic and local administration of interferon-gamma impairs wound healing. Zhonghua Zheng Xing Shao Shang Wai Ke Za Zhi. 1995;11:209-211.

38. Hillan K, Nordlinger B, Ballet F, Puts JP, Infante R. The healing of colonic anastomoses after early intraperitoneal chemotherapy: an experimental study in rats. J Surg Res. 1988;44:166-171.

39. Ersoy E, Akbulut H, Moray G. Effects of oxaliplatin and 5-fluorouracil on the healing of colon anastomoses. Surg Today. 2009;39:38-43.

40. Graf W, Weiber S, Jiborn H, Píhlman L, Glimelius B, Zederfeldt $\mathrm{B}$. The roles of nutritional depletion and drug concentration in 5-fluorouracil-induced inhibition of colonic healing. J Surg Res. 1994;56:452-456.

41. Weiber S, Graf W, Glimelius B, Jiborn H, PÍhlman L, Zederfeldt $\mathrm{B}$. The effect of 5-fluorouracil on wound healing and collagen synthesis in left colon anastomoses. An experimental study in the rat. Eur Surg Res. 1994;26:173-178.
42. Weiber S, Graf W, Glimelius B, Jiborn H, PÍhlman L, Zederfeldt B. Experimental colonic healing in relation to timing of 5-fluorouracil therapy. Br J Surg. 1994;81:1677-1680.

43. Kanellos I, Kazantzidou D, Evangelou I, Galovatsea K, Zaraboukas T, Dadoukis I. Healing of colonic anastomoses after immediate and delayed administration of 5-fluorouracil plus folinic acid. Eur Surg Res. 1998; 30:312-317.

44. Kanellos I, Odisseos C, Kazantzidou D, Galovatsea K, Zaraboukas T, Dadoukis I. Effect of early versus delayed postoperative injection of 5-fluorouracil plus interferon-alpha on colonic healing. Eur Surg Res. 1998;30:414-418.

45. Nayci A, Cakmak M, Renda N, Aksoyek S, Yucesan S. Effect of electromagnetic fields and early postoperative 5-fluorouracil on the healing of colonic anastomoses. Int J Colorectal Dis. 2003;18:136-141.

46. Stoop MJ, Dirksen R, Wobbes T, Hendriks T. Effects of early postoperative 5-fluorouracil and ageing on the healing capacity of experimental intestinal anastomoses. Br J Surg. 1998;85:1535-1538.

47. el-Malt M, Ceelen W, Van den Broecke C et al. Influence of neo-adjuvant chemotherapy with 5-fluorouracil on colonic anastomotic healing: experimental study in rats. Acta Chir Belg. 2003;103:309-314.

48. Kuzu MA, Köksoy C, Kale T, Demirpençe E, Renda N. Experimental study of the effect of preoperative 5-fluorouracil on the integrity of colonic anastomoses. Br J Surg. 1998;85: 236-239.

49. Kim CS, Buchmiller TL, Fonkalsrud EW, Phillips JD. The effect of anabolic steroids on ameliorating the adverse effects of chronic corticosteroids on intestinal anastomotic healing in rabbits. Surg Gynecol Obstet. 1993;176:73-79.

50. Polat A, Nayci A, Polat G, Aksöyek S. Dexamethasone downregulates endothelial expression of intercellular adhesion molecule and impairs the healing of bowel anastomoses. Eur J Surg. 2002;168:500-506.

51. Mantzoros I, Kanellos I, Demetriades H et al. Effects of steroid on the healing of colonic anastomoses in the rat. Tech Coloproctol. 2004;8(Suppl 1):180-183.

52. Eubanks TR, Greenberg JJ, Dobrin PB, Harford FJ, Gamelli RL. The effects of different corticosteroids on the healing colon anastomosis and cecum in a rat model. Am Surg. 1997;63:266-269. 